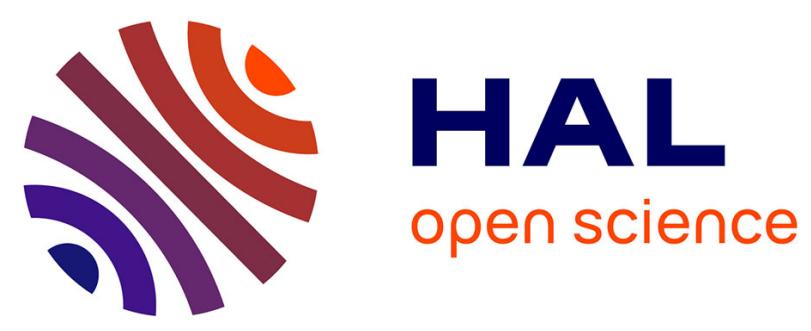

\title{
Attosecond chirp-encoded dynamics of light nuclei Attosecond chirp-encoded dynamics of light nuclei
}

\author{
Stefan Haessler, W Boutu, M Stankiewicz, L J Frasinski, Sébastien J. Weber,
} J Caillat, R Taïeb, A Maquet, P Breger, P Monchicourt, et al.

\section{- To cite this version:}

Stefan Haessler, W Boutu, M Stankiewicz, L J Frasinski, Sébastien J. Weber, et al.. Attosecond chirp-encoded dynamics of light nuclei Attosecond chirp-encoded dynamics of light nuclei. Journal of Physics B: Atomic and Molecular Physics, 2009, 42, pp.134002. 10.1088/0953-4075/42/13/134002 . hal-01164781

\section{HAL Id: hal-01164781 \\ https://hal-ensta-paris.archives-ouvertes.fr/hal-01164781}

Submitted on 18 Jun 2015

HAL is a multi-disciplinary open access archive for the deposit and dissemination of scientific research documents, whether they are published or not. The documents may come from teaching and research institutions in France or abroad, or from public or private research centers.
L'archive ouverte pluridisciplinaire HAL, est destinée au dépôt et à la diffusion de documents scientifiques de niveau recherche, publiés ou non, émanant des établissements d'enseignement et de recherche français ou étrangers, des laboratoires publics ou privés. 


\title{
Attosecond chirp-encoded dynamics of light nuclei
}

\author{
S Haessler $^{1}$, W Boutu ${ }^{1} \ddagger$, M Stankiewicz ${ }^{2}$, L J Frasinski ${ }^{3}$, S \\ Weber $^{1} \S$, J Caillat ${ }^{4}, \mathbf{R}$ Taïeb $^{4}$, A Maquet ${ }^{4}, \mathbf{P}$ Breger $^{1}, \mathbf{P}$ \\ Monchicourt $^{1}$, B Carré ${ }^{1}$ and $\mathrm{P}$ Salières $^{1}$ \\ 1 CEA Saclay, IRAMIS, Service des Photons, Atomes et Molécules, 91191 \\ Gif-sur-Yvette, France. \\ 2 Institute of Physics, Jagiellonian University, ul. Reymonta 4, 30-059 Kraków, \\ Poland. \\ 3 The Blackett Laboratory, Imperial College London, South Kensington Campus, \\ London SW7 2AZ, United Kingdom. \\ 4 UMPC Université Paris 06, CNRS, UMR 7614, LCPMR, 11 rue Pierre et Marie \\ Curie, 75231 Paris Cedex 05, France. \\ E-mail: pascal.salieres@cea.fr
}

\begin{abstract}
We study the spectral phase of high-order harmonic emission as an observable for probing ultrafast nuclear dynamics after ionization of a molecule. Using a strong field approximation theory that includes nuclear dynamics, we relate the harmonic phase to the phase of the overlap integral of the nuclear wavefunctions of the initial neutral molecule and the molecular ion after an attosecond probe delay. We determine experimentally the group delay of the high harmonic emission from $\mathrm{D}_{2}$ and $\mathrm{H}_{2}$ molecules, which allows us to verify the relation between harmonic frequency and the attosecond delay. The small difference in the harmonic phase between $\mathrm{H}_{2}$ and $\mathrm{D}_{2}$ calculated theoretically is consistent with our experimental results.
\end{abstract}

Submitted to: J. Phys. B: At. Mol. Phys.

\section{Introduction}

Dynamic imaging of molecular structure with ångström space and attosecond time resolution is one of the most exciting recent developments in attosecond science. The attosecond timescale is inherent to intra-atomic/molecular electron dynamics and to the movement of the lightest nuclei. The ultimate goal of this research might be tomographic imaging [1] of an evolving molecular orbital. A challenging goal in chemical physics is to trace ultrafast nuclear re-arrangements or vibrations. In particular the transfer

$\ddagger$ Present address: Department of Chemistry, University of California, Berkeley, California 94720, USA and Chemical Sciences Division, Lawrence Berkeley National Laboratory, Berkeley, California 94720, USA.

$\S$ Present address: CNRS, Université de Toulouse, UPS, Laboratoire Collisions Agrégats Réactivité, IRSAMC, France. 
of protons within large molecules plays an important role in many chemical reactions relevant to life sciences [2, 3], combustion and astrophysics [4]. A great deal of research has thus been devoted to the observation of such nuclear re-arrangements achieving femtosecond $[5,6,7]$ or attosecond $[8,9]$ time-resolution.

A technically fairly simple, yet powerful method termed PACER (probing attosecond dynamics by chirp encoded recollision) has been proposed for observing the expansion of a vibrating hydrogen molecular ion immediately after ionization. It was studied first numerically [10] and then demonstrated experimentally two years ago [9]. Briefly, the ratio of high harmonic spectra generated under the same conditions in protonated and deuterated species of a molecule is measured, and the nuclear dynamics are retrieved by means of a genetic algorithm. The three steps, commonly used to describe high harmonic generation (HHG) $[11,12]$, are considered as a pump, a delay-stage, and a probe process: A strong laser field ionizes the molecule, launching simultaneously an electron wavepacket of attosecond duration into the continuum and a time dependent nuclear wavepacket onto the electronic ground state potential surface of the molecular ion. The continuum electron wavepacket is subsequently accelerated and driven back to the ion by the laser field, while the nuclear wavepacket evolves in parallel. At recollision, there is a certain probability for recombination to the ground state, releasing the accumulated kinetic energy of the electron in the form of an attosecond burst of XUV light. For coherent emission, recombination has to lead back to the initial state, the nuclear part of which is the vibrational ground state of the neutral molecule. The probability of this transition depends on the overlap of this ground state nuclear wavefunction with the evolved nuclear wavepacket of the molecular ion at the recombination time, i.e. recombination will be all the less likely the further the ion has evolved. Only this coherent light will be detectable as macroscopic signal produced by many emitters. Obviously, recombination can lead back to an excited state of the molecule, like an electronic state with the same symmetry as the initial state, or a vibrationally excited state. This excited state would, however, have an arbitrary phase relative to the continuum electron, which is 'phase locked' to the ground state and the light emission would consequently be incoherent, adding only a weak background to the high harmonic emission.

Scanning the pump-probe delay is achieved by the inherent chirp of the recolliding electron wavepacket: for the short electron trajectories, higher harmonics are associated with larger electron excursion times and thus larger recollision times. In [9], this chirp was assumed to be the same as for rare-gas atoms, as measured in [13]. The latter study showed that the measured recollision times are very well described by the Lewenstein model [14]. Recently, it has been shown that the atomic and molecular recollision times may be equal provided that (i) the nuclear movement can be neglected on a sub-lasercycle timescale (which is true for heavier molecules such as $\mathrm{N}_{2}$ and $\mathrm{CO}_{2}$ ), (ii) the sample is unaligned [15] or aligned in such a way that two-centre interference [16] does not occur, and (iii) the two species have the same ionization potential [17].

The aim of this work is two-fold: Firstly, using the RABITT (Reconstruction of 
Attosecond Beating by Interference of Two-photon Transitions)[18, 19] technique, we want to verify experimentally for $\mathrm{H}_{2}$ and $\mathrm{D}_{2}$ molecules that the frequency-time mapping, which is at the heart of the PACER method, is not affected by the molecular ion's expansion. Secondly, and more generally, we will address the question how the rapid nuclear dynamics affect the phase of the high harmonic emission and whether it could be linked more directly to the nuclear dynamics than the harmonic intensity. The second of these points has been studied in [20] using a different experimental technique, which will be discussed and compared to our experimental findings.

In our paper, we first review the theoretical basis of PACER in section 2.1, followed by a detailed analysis of the result with regard to the phase differences between $\mathrm{H}_{2}$ and $\mathrm{D}_{2}$ in section 2.2. We then present our experimental configuration and results in sections 3 and 4, respectively. Finally, we draw conclusions of our study in section 5 .

\section{Theoretical description}

\subsection{Overview of the strong-field approximation theory including nuclear dynamics}

The theoretical basis for PACER is thoroughly described in $[10,21]$ but to address the above questions we will briefly review the essential points. The two wavepackets launched in the ionization step of the three step model are correlated and their evolution is followed in [10] by considering the simplest case of $\mathrm{H}_{2}$ and $\mathrm{D}_{2}$ molecules and solving numerically the time-dependent Schrödinger equation (TDSE), including the interaction of the single active electron with the screened nuclei, i.e. non-Born-Oppenheimer couplings. The authors then derive a modified Lewenstein model [14], also called the strong-field approximation (SFA) model, incorporating the nuclear motion within the Born-Oppenheimer (BO) approximation. The results of this model agree very well with the exact TDSE calculation, justifying the approximations made for the considered parameters $\left(780 \mathrm{~nm}, 6\right.$-cycle pulse with $\left.4 \times 10^{14} \mathrm{~W} / \mathrm{cm}^{2}\right)$. It is hardly possible to rigorously deduce a range of laser parameters where the SFA and BO approximation are valid. The SFA approach has been extensively compared with TDSE calculations and experiments in rare-gas atoms, and was shown to be in satisfactory agreement for a Keldysh parameter $\gamma \leq 1$. The same should hold for the simplest molecules like $\mathrm{H}_{2}$. The BO approximation on the other hand obviously becomes questionable for the lightest nuclei and indeed there are deviations observed, e.g. from the Franck-Condon principle for the tunnel ionization step [22, 23].

Following the assumptions of the SFA and assuming that only one electron can become active, one makes an ansatz for the full wavefunction (atomic units are used unless stated otherwise):

$$
\begin{aligned}
\psi\left(\boldsymbol{r}_{1}, \boldsymbol{r}_{2}, R, t\right)=\mathrm{e}^{-i E_{0} t}\left\{\chi_{0}(R) \psi_{R}\left(\boldsymbol{r}_{1}, \boldsymbol{r}_{2}\right)\right. \\
\left.+\int \frac{\mathrm{d}^{3} k}{(2 \pi)^{3}} \phi(\boldsymbol{k}, R, t)\left[\exp \left(\mathrm{i} \boldsymbol{k} \cdot \boldsymbol{r}_{1}\right) \psi_{R}^{+}\left(\boldsymbol{r}_{2}\right)+\exp \left(\mathrm{i} \boldsymbol{k} \cdot \boldsymbol{r}_{2}\right) \psi_{R}^{+}\left(\boldsymbol{r}_{1}\right)\right]\right\},
\end{aligned}
$$

where $\boldsymbol{r}_{1}, \boldsymbol{r}_{2}$ are the coordinates of the two electrons, $\chi_{0}(R) \psi_{R}\left(\boldsymbol{r}_{1}, \boldsymbol{r}_{2}\right)$ is the real-valued 
ground state wavefunction of $\mathrm{H}_{2}$ (or its isotopes) in the $\mathrm{BO}$ approximation, i.e. written as a product of a nuclear part $\chi_{0}$ and an electronic part $\psi_{R}$, and $\psi_{R}^{+}\left(\boldsymbol{r}_{1 / 2}\right)$ is the electronic ground-state $\mathrm{BO}$ wave function of $\mathrm{H}_{2}^{+} . \phi(\boldsymbol{k}, R, t)$ describes the time-dependence of the continuum electron as well as the nuclear part of the $H_{2}^{+}$wave function. $E_{0}$ is the ground state energy eigenvalue. We choose the origin of energy such that the lowest BO potential of $\mathrm{H}_{2}^{+}, V_{\mathrm{BO}}^{+}(R)$, takes the value zero at the average internuclear distance $\bar{R}_{0}$ in the vibrational ground state of the neutral molecule. We thus subtract $V_{\mathrm{BO}}^{+}\left(\bar{R}_{0}\right)$ from all curves, which consequently replaces $E_{0}$ in $(1)$ by $E_{0}-V_{\mathrm{BO}}^{+}\left(\bar{R}_{0}\right)=-I_{\mathrm{p}}$, where $I_{\mathrm{p}}$ is an effective vertical ionization potential.

The TDSE for an $\mathrm{H}_{2}$ molecule with fixed orientation, driven by a linearly polarized laser field $E(t)$ along the x-axis is then solved analytically, neglecting non-BO couplings and laser field interaction with $\psi_{R}^{+}$, leading to an expression for $\phi(\boldsymbol{k}, R, t)$ that allows calculating the time-dependent dipole momentum along $\mathrm{x}$ :

$$
\begin{aligned}
V_{x}(t)= & -\mathrm{i}\left\langle\psi(t)\left|\partial_{x_{1}}+\partial_{x_{2}}\right| \psi(t)\right\rangle \\
= & 2 \mathrm{i} \int_{0}^{t} \mathrm{~d} t^{\prime} \int \frac{\mathrm{d}^{3} p}{(2 \pi)^{3}} E\left(t^{\prime}\right) \bar{d}_{\mathrm{i}}\left[\boldsymbol{p}+\boldsymbol{A}\left(t^{\prime}\right)\right] \exp \left[\mathrm{i} S\left(\boldsymbol{p}, t, t^{\prime}\right)\right] \\
& \times \int \mathrm{d} R \chi_{0}(R) v_{\mathrm{r}}[\boldsymbol{p}+\boldsymbol{A}(t), R] \chi\left(R, t-t^{\prime}\right)+\text { c.c. },
\end{aligned}
$$

where

$$
S\left(\boldsymbol{p}, t, t^{\prime}\right)=-\int_{t^{\prime}}^{t} \mathrm{~d} t^{\prime \prime}\left\{\frac{\left[\boldsymbol{p}+\boldsymbol{A}\left(t^{\prime \prime}\right)\right]^{2}}{2}+I_{\mathrm{p}}\right\}
$$

is the semiclassical action of the continuum electron, $\boldsymbol{p}=\boldsymbol{k}-\boldsymbol{A}(t)$ is its canonical momentum and $\boldsymbol{A}(t)=-\int_{-\infty}^{t} \boldsymbol{E}\left(t^{\prime}\right) \mathrm{d} t^{\prime}$ is the vector potential of the laser field. The evolution of the nuclear wavepacket $\chi\left(R, t-t^{\prime}\right)$ in the molecular ion is obtained by solving the Schrödinger equation

$$
\mathrm{i} \dot{\chi}(R, t)=\left[-\partial_{R}^{2} / M+V_{\mathrm{BO}}^{+}(R)\right] \chi(R, t),
$$

where $M$ is the mass of one nucleus. For the initial condition, the Franck-Condon principle is applied: $\chi\left(R, t^{\prime}\right)=\chi_{0}(R)$. This is a good approximation for laser intensities on the order of $10^{14} \mathrm{~W} / \mathrm{cm}^{2}$ and molecules aligned perpendicular to the laser polarization direction [22, 23], and should thus also hold for an unaligned molecular sample. Note, that the interaction with the strong laser field is neglected in (4) although the laser field significantly modifies the potential curve the nuclear wavepacket moves on and may couple different electronic states. The effect, however, is strongest at internuclear separations $R$ of the molecular ion above its equilibrium value $R_{0}=2$ a.u. and in particular close to the avoided crossings of the field dressed $\sigma_{\mathrm{g}}$ and $\sigma_{\mathrm{u}}$ states of the molecular ion around $R=4$ a.u., leading to bond-softening and bond-hardening [24, 25]. Within the time window covered by the excursion times in HHG with an $800 \mathrm{~nm}$ laser $(<1.8 \mathrm{fs})$, the nuclear wavepacket cannot evolve very far and in fact still remains on the falling edge of the $\sigma_{\mathrm{g}}$ potential curve. At these $R$, the potential is barely affected (see figure 13 in [24], where $\mathrm{H}_{2}^{+}$potentials are shown for an intensity of $1 \times 10^{14} \mathrm{~W} / \mathrm{cm}^{2}$ ) and the field-free calculation using (4) should be valid for the nuclear wavepacket evolution 
accessible in the experiment. The effect of laser dressing on the outcome of PACER-like experiments has also been studied theoretically by Chirilă and Lein [26] and found to be negligible for $800 \mathrm{~nm}$ lasers due to the short time between ionization and recombination. For mid-IR lasers (e.g. 1.5 or $2 \mu \mathrm{m}$ ), however, dressing leads to significant modifications of the nuclear dynamics.

In expression (2) for the dipole momentum, the three steps described in the introduction are recovered as follows:

(i) At time $t^{\prime}$ the active electron tunnels into the continuum with a probability amplitude $E\left(t^{\prime}\right) \bar{d}_{\mathrm{i}}$. Here, a simplification has been introduced: the $R$-dependence of the matrix element $d_{i}$ has been neglected and instead the effective matrix element

$$
\bar{d}_{\mathrm{i}}(\boldsymbol{k})=\left(\int\left|\left\langle\exp \left(\mathrm{i} \boldsymbol{k} \cdot \boldsymbol{r}_{1}\right) \psi_{R}^{+}\left(\boldsymbol{r}_{2}\right)\left|x_{1}\right| \psi_{R}\left(\boldsymbol{r}_{1}, \boldsymbol{r}_{2}\right)\right\rangle \chi_{0}(R)\right|^{2} \mathrm{~d} R\right)^{1 / 2},
$$

averaged over all possible values of the internuclear distance $R$, is used. (ii) The active electron moves in the continuum under the influence of the laser field only, until time $t$, acquiring a phase relative to the ground state of $S\left(\boldsymbol{p}, t, t^{\prime}\right)$. (iii) At time $t$, the continuum electron recombines with the molecular ion to the neutral ground state, the amplitude of which is given by

$\nu(\boldsymbol{k})=-\mathrm{i} \int \chi_{0}(R)\left\langle\psi_{R}\left(\boldsymbol{r}_{1}, \boldsymbol{r}_{2}\right)\left|\partial_{x_{1}}\right| \exp \left[\mathrm{i} \boldsymbol{k} \cdot \boldsymbol{r}_{1}\right] \psi_{R}^{+}\left(\boldsymbol{r}_{2}\right)\right\rangle \chi\left(R, t-t^{\prime}\right) \mathrm{d} R$.

Note that the Bra-Ket matrix element implies integration over both $\boldsymbol{r}_{1}$ and $\boldsymbol{r}_{2}$ and that its functional form is identical for $\mathrm{H}_{2}$ and $\mathrm{D}_{2}$. It is still parametrically $R$-dependent and is written here for a specific alignment angle $\theta$ of the molecular axis with respect to the laser polarization. Adopting a linear combination of atomic orbitals (LCAO) for the electronic ground states of the neutral molecule and the molecular ion with $\psi_{R}=\psi_{R}^{+}\left(\boldsymbol{r}_{1}\right) \psi_{R}^{+}\left(\boldsymbol{r}_{2}\right)$ [21], one can reduce its $R$-dependence to an interference kernel $\cos (k R \cos (\theta) / 2)$. Any possible difference between the recombination amplitudes for $\mathrm{H}_{2}$ and $\mathrm{D}_{2}$ with an alignment distribution $\sigma(\theta)$ must then be contained in

$$
c(k, R)=\int \sigma(\theta) \int \chi_{0}(R) \cos (k R \cos (\theta) / 2) \chi\left(R, t-t^{\prime}\right) \mathrm{d} R \mathrm{~d} \theta .
$$

The interference kernel is real valued and will change sign at $k_{0}=\pi /[R \cos (\theta)]$, which translates to a sudden $\pi$ phase jump. For $\mathrm{H}_{2}$ molecules partly aligned along the laser polarization direction, two-centre interference will indeed play a role when using an $800 \mathrm{~nm}$ laser [27]. Detecting the phase jump at some harmonic order $q$ associated to the excursion time $\tau_{q}$, as was recently demonstrated for $\mathrm{CO}_{2}$ molecules [17], would be an observation of dynamic two-centre interference [27] on the observable "harmonic phase". This would provide a means of observing when the centre of mass $\tilde{R}\left(t-t^{\prime}\right)$ of the product of the nuclear wavefunctions $\chi_{0}(R)$ and $\chi\left(R, t-t^{\prime}\right)$ passes through the value $\tilde{R}=\pi /\left[\sqrt{2 q \omega_{0}} \cos \left(\theta_{\mathrm{m}}\right)\right]$ satisfying the destructive interference condition, where $\theta_{\mathrm{m}}$ is the modal value of the alignment distribution $\sigma(\theta)$ and $\omega_{0}$ is the driving laser frequency. For $\mathrm{D}_{2}$ molecules, this dynamic two-centre interference then occurs at a larger time and hence larger harmonic order. This would, however, only provide a measurement of one 
point $\tilde{R}\left(\tau_{q}\right)$ for each isotope and does not directly allow to continuously trace the nuclear dynamics.

For randomly aligned molecules, the $\theta$-integral in (7) reduces to $\sin (k R / 2) /(k R / 2)$, the sign change of which would occur at too high harmonic orders to be observed using an $800 \mathrm{~nm}$ laser. Dropping the interference kernel thus still gives a reasonably good approximation for unaligned molecules [9]. This has the great advantage of reducing the $R$-dependence of ( 7$)$ to a $k$-independent nuclear overlap integral

$$
C\left(t-t^{\prime}\right)=\int \chi_{0}(R) \chi\left(R, t-t^{\prime}\right) \mathrm{d} R \text {. }
$$

The intensity of a harmonic that is dominated by a single excursion time $\tau=t-t^{\prime}$ is therefore proportional to $|C(\tau)|^{2}$, which monotonically decreases with time when the molecular ion expands. The decrease is obviously slower in the heavier isotope since the nuclear dynamics is slower. When the contribution of the short trajectories is isolated in the experiment, the measured ratio of harmonic intensities for $\mathrm{D}_{2}$ and $\mathrm{H}_{2}$, being proportional to $\left|C^{\mathrm{D}_{2}}(\tau) / C^{\mathrm{H}_{2}}(\tau)\right|^{2}$, will thus monotonically increase with harmonic order. This has been experimentally confirmed $[9,20]$.

\subsection{Analysis of phase effects}

The three steps of the high harmonic emission process influence the phase of the radiation emitted in the following manner:

(i) The term (5) is essentially the same for the two isotopes, since the electronic wavefunctions $\psi_{R}$ and $\psi_{R}^{+}$are identical. There is a small difference in the width of the initial nuclear wavepackets $\chi_{0}(R)$, but as they are purely real valued this will not translate to a phase difference.

(ii) In (3), the ionization potential for $\mathrm{D}_{2}$ is in fact about $40 \mathrm{meV}$ higher than for $\mathrm{H}_{2}$ [28], but this difference is negligible. At this point, Kanai et al.[20] introduced to the action an ionization potential being a function of the internuclear distance $R\left(t^{\prime \prime}\right)$, which then gave a different contribution for $\mathrm{H}_{2}$ and $\mathrm{D}_{2}$ due to the different nuclear dynamics. They described this term as "the interaction between the molecular ion and the electron". We think such a modification of the action is incorrect, because any ion-electron interaction is explicitly excluded in the SFA and thus cannot appear in the result. Note, that the influence of the ion's Coulomb potential has been shown to be noticeable only for the lowest harmonics with energies close to $I_{\mathrm{p}}$ [29]. Secondly, the $I_{\mathrm{p}}$ in the action is nothing else but the energy eigenvalue of the neutral molecule BO ground state after a recalibration of the energy axis. This ground state is stationary and its energy does not change with time. We thus conclude that, within the framework of the SFA, the continuum electron dynamics is not only the same in $\mathrm{H}_{2}$ and $\mathrm{D}_{2}$ but it is also the same as in an atom of equal ionization potential. It is thus perfectly justified to use the frequency-time mapping as calculated from the three-step model as was done in the experiments $[9,27]$. 


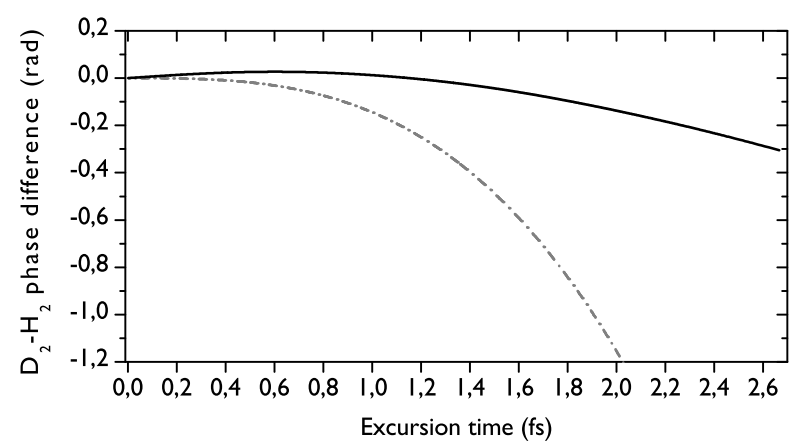

Figure 1. Theoretical phase difference from a full quantum mechanical calculation (full line) and from the quasiclassical expression (9) (dash-dotted line).

(iii) We have now established that the recolliding continuum electron wavepacket is, within the framework of the SFA, identical in the cases of $\mathrm{H}_{2}$ and $\mathrm{D}_{2}$. Along the lines proposed by Itatani et al.[1], we can now directly obtain the phase difference of the harmonic emissions from the two isotopes from the phase difference of the recombination amplitudes $\nu(\boldsymbol{k})$ given in (6). As discussed earlier, when neglecting twocentre interference, the difference between $\mathrm{H}_{2}$ and $\mathrm{D}_{2}$ is contained in (8). It is thus the phase of the integral $C(\tau)$ that is passed on to the harmonic emission and potentially contains a signature of the nuclear dynamics. As $\chi_{0}(R)$ is real-valued, the evolved nuclear wavepackets $\chi(R, \tau)$ are the only remaining source of phase difference.

We first treat the nuclear evolution classically and hence take $R$ as a sharply defined variable depending on the excursion time $\tau$. The nuclei only convert potential energy $V_{\mathrm{BO}}^{+}(R)$ into kinetic energy $T_{\mathrm{n}}$. Using $V_{\mathrm{BO}}^{+}\left(\bar{R}_{0}\right)=0$, the Lagrangian reads $\mathcal{L}(\tau)=T_{\mathrm{n}}-V_{\mathrm{BO}}^{+}=-2 V_{\mathrm{BO}}^{+}[R(\tau)]$. We can thus write the quasiclassical action of the nuclei in the molecular ion as $S_{\mathrm{n}}\left(t-t^{\prime}\right)=-2 \int_{t^{\prime}}^{t} \mathrm{~d} t^{\prime \prime} V_{\mathrm{BO}}^{+}\left[R\left(t^{\prime \prime}\right)\right]$. The quasiclassical estimate for the harmonic phase difference $\Delta \varphi_{\mathrm{qcl}}^{\mathrm{D}_{2}-\mathrm{H}_{2}}$ between $\mathrm{D}_{2}$ and $\mathrm{H}_{2}$ is the difference of the quasiclassical actions $S_{\mathrm{n}}$ of their evolving nuclear wavepackets. Linearizing the potential curve of the ion around the average internuclear distance $\bar{R}_{0}$ of the ground state, i.e. $V_{\mathrm{BO}}^{+}(R)=\left.\partial_{R} V_{\mathrm{BO}}^{+}(R)\right|_{\bar{R}_{O}} \Delta R$, approximating the nuclear movement by classical uniformly accelerated motion: $\Delta R^{\mathrm{H}_{2} / \mathrm{D}_{2}}(\tau)=-\left.\partial_{R} V_{\mathrm{BO}}^{+}(R)\right|_{\bar{R}_{O}} /\left(2 M^{\mathrm{H}, \mathrm{D}}\right) \tau^{2}$, and using $M^{\mathrm{D}}=2 M^{\mathrm{H}}$, we find the simple relation

$$
\Delta \varphi_{\mathrm{qcl}}^{\mathrm{D}_{2}-\mathrm{H}_{2}}(\tau)=\Delta S_{\mathrm{n}}^{\mathrm{D}_{2}-\mathrm{H}_{2}}=\left.\partial_{R} V_{\mathrm{BO}}^{+}(R)\right|_{\bar{R}_{O}} \Delta R^{\mathrm{H}_{2}}(\tau) \frac{\tau}{3} .
$$

The slope of the $\mathrm{BO}$ potential of the molecular ion at $\bar{R}_{0}=1.4$ a.u. is $\left.\partial_{R} V_{\mathrm{BO}}^{+}(R)\right|_{\bar{R}_{O}}=$ -0.15 a.u.. Equation (9) turns out to be - up to a factor of 2 - the same as the phase difference calculated by Kanai et al. [20]. The difference is that we identify its origin as the phase of the evolved nuclear wavepacket in the molecular ion and conclude that it only comes into play during the recombination step, whereas the continuum electron dynamics remain unaffected.

Nevertheless, this quasiclassical approach allows us to find only a rough estimate. Recombination and thus high harmonic emission are only possible as long as there is an overlap of $\chi_{0}(R)$ and $\chi(R, \tau)$. The quantum nature of the nuclear movement, i.e. the 
finite width of the nuclear wavefunctions, is thus absolutely essential. Equation (9) can only give an upper limit for the phase difference between the harmonic emissions from the two isotopes, because, roughly speaking, the nuclear position where the product $\chi_{0}(R) \chi(R, \tau)$ is maximum evolves considerably slower than the classical trajectory of the nuclei in the ion. For a reliable result, we thus have to calculate $C(\tau)$ exactly. Since the Hamiltonian in (4) is time-independent, it is fairly straightforward to compute the evolution of $\chi(R, \tau)$. Once eigenstates $\chi_{n}(R)$ and eigenenergies $E_{n}$ of the Hamiltonian are found, one can project the initial wavepacket $\chi(R, 0)=\chi_{0}(R)$ onto this basis and calculate $\chi(R, \tau)=\sum_{n} \exp \left[-\mathrm{i} E_{n} \tau\right] a_{n} \chi_{n}(R)$ with $a_{n}=\left\langle\chi_{n} \mid \chi_{0}\right\rangle$. We have done this calculation for $n$ up to 105, which has given us the complex $C(\tau)$ for $\mathrm{H}_{2}$ and $\mathrm{D}_{2}$ according to (8), i.e. neglecting two-centre interference. We checked that our ratio $\left|C^{\mathrm{D}_{2}}(\tau) / C^{\mathrm{H}_{2}}(\tau)\right|^{2}$ (shown in figure 3 ) agrees very well with the one by Lein $[9,10]$. In figure 1, we compare the $\mathrm{D}_{2}-\mathrm{H}_{2}$ phase difference of the $C(\tau)$ obtained from this full calculation with the phase difference $\Delta \varphi_{\mathrm{qcl}}^{\mathrm{D}_{2}-\mathrm{H}_{2}}(\tau)$ from the quasiclassical treatment. Obviously, the quasiclassical result is a gross over-estimation and only the full quantum mechanical treatment is adequate.

\section{Experimental configuration}

The experiments were carried out using the LUCA laser at CEA Saclay, delivering up to $30 \mathrm{~mJ}$ in $55 \mathrm{fs}$ pulses at $20 \mathrm{~Hz}$ repetition rate. The setup is shown in Fig. 2 in [13]. A supersonic gas jet provides the molecular sample with an effective medium length of $\approx 1 \mathrm{~mm}$. The Mach-Zehnder type interferometer for the $\operatorname{RABITT}[18,19]$ measurement is based on drilled mirrors separating the laser beam in two parts: an annular generating beam (outer diameter cut to $17 \mathrm{~mm}$ by an iris) and a central probe beam (diameter $\approx 4 \mathrm{~mm}$ ). The latter can be delayed by a piezoeletric translation stage with interferometric stability. Both beams are then collinearly focused by a $1 \mathrm{~m}$ focal length lens $\approx 5 \mathrm{~mm}$ before the gas jet, making sure that the short trajectory contribution to the HHG emission is selected by phase matching [30]. In the gas jet, the intensities of generating and probe beam are $1.2 \times 10^{14} \mathrm{~W} \mathrm{~cm}^{-2}$ and $\sim 10^{11} \mathrm{~W} \mathrm{~cm}^{-2}$, respectively. An iris then blocks the annular generating beam, whereas the high harmonics together with the on-axis probe beam are focused by a broad-band Au-coated toroidal mirror into an effusive neon gas jet. A magnetic-bottle electron spectrometer (MBES) [31] detects the photoelectron spectrum. With the weak IR probe pulse present, spectral sidebands occur, created by two-photon ionization involving a high harmonic photon and an IR photon. Analysis of these sidebands as a function of the probe beam delay gives access to the relative phase of two successive harmonic orders $\varphi_{q+2}-\varphi_{q}$, and thus to the group delay [13]:

$$
\tau_{\mathrm{g}}\left(\omega_{q+1}\right):=\left.\frac{\partial \varphi}{\partial \omega}\right|_{q+1} \approx \frac{\varphi_{q+2}-\varphi_{q}}{2 \omega_{0}} .
$$

Note that the small modulation of the HHG intensity due to interference with the probe beam in the generating medium allows us to determine absolute group delay values with 


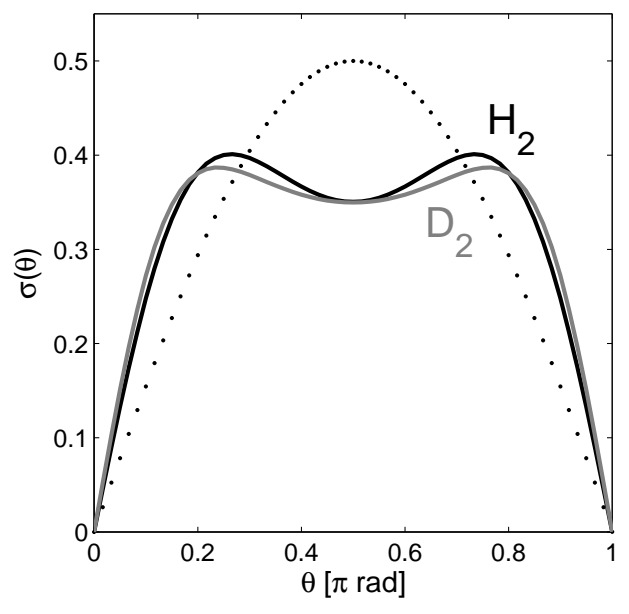

Figure 2. Calculated alignment distributions $\sigma(\theta)$ at the generating pulse peak for our experimental conditions. Black and grey lines show $\sigma(\theta)$ for $\mathrm{H}_{2}$ and $\mathrm{D}_{2}$, respectively. The dotted line shows an isotropic distribution for comparison.

respect to the generating laser field maximum [32].

We verified that partial alignment induced by the generating pulse is negligible by calculating the angular distributions $\sigma(\theta)$ at the peak of the pulse by the method described in the appendix of [33], using the 4th order Runge-Kutta method [34] for the TDSE integration. We use a $\sin ^{2}$ pulse of 55 fs FWHM duration and a temperature of $90 \mathrm{~K}$ for $\mathrm{D}_{2}$ and $150 \mathrm{~K}$ for $\mathrm{H}_{2}$ due to the different backing pressures necessary to achieve equal gas densities. The result is shown in figure 2. Obviously the alignment distributions are modified during the generating pulse but not strongly enough for dynamic two-centre interference [27] to play a role.

\section{Experimental Results}

Using the RABITT technique, we have measured intensity and group delay of the high harmonic emission from unaligned $\mathrm{H}_{2}$ and $\mathrm{D}_{2}$ molecules $\left(I_{\mathrm{p}}^{\mathrm{H}_{2} / \mathrm{D}_{2}}=15.43 \mathrm{eV}\right)$ as well as for $\mathrm{Ar}$ atoms, which have a very similar ionization potential $\left(I_{\mathrm{p}}^{\mathrm{Ar}}=15.76 \mathrm{eV}\right)$. We were able to measure the group delay for harmonic orders between 15 to 31 and obtain harmonic intensities from photoelectron spectra recorded without the IR probe beam present for orders 17 to 33 . We had no means of directly measuring the density in the generating gas jet. As the efficiency of turbo molecular pumps rapidly drops with decreasing molecular weight, the residual pressure in the vacuum chambers was rather high when generating harmonics in $\mathrm{D}_{2}$ and even more so for $\mathrm{H}_{2}$. We first determined the maximum possible gas jet backing pressure for $\mathrm{H}_{2}$ by observing its characteristic trace in the recorded photoelectron spectra. With a significant density of $\mathrm{H}_{2}$ molecules having migrated about $2 \mathrm{~m}$ down to the interaction region of the MBES and mixing with the injected neon, the peaks in the photoelectron spectrum are broadened on the red side with a substructure corresponding to the vibrational levels of $\mathrm{H}_{2}^{+}$ions. We then 


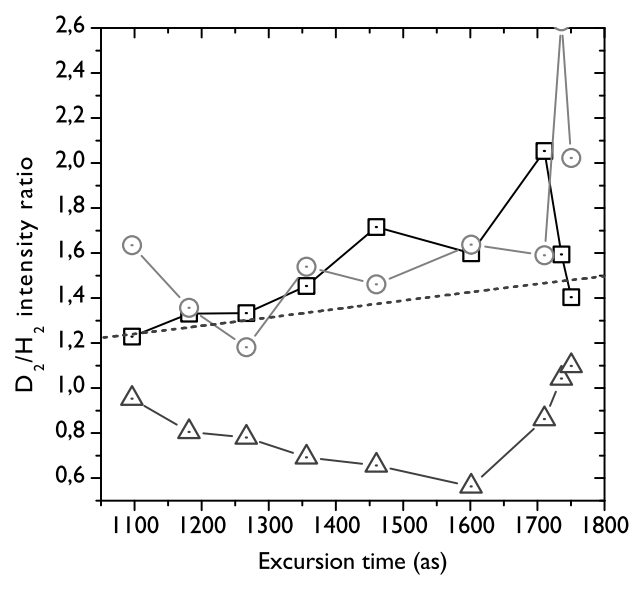

Figure 3. $\mathrm{D}_{2} / \mathrm{H}_{2}$ intensity ratios for harmonics 17 to 33 measured with different gas pressures. Residual pressure readings $p_{\mathrm{H}_{2}}=p_{\mathrm{D}_{2}}=5 \times 10^{-2}$ mbar in the HHG chamber $(\square), p_{\mathrm{H}_{2}}=5 \times 10^{-2}$ mbar, $p_{\mathrm{H}_{2}} / p_{\mathrm{D}_{2}}=1.3$ and the $\mathrm{D}_{2}$ spectrum has been corrected by multiplication with $(1.3)^{2}(\mathrm{O}), p_{\mathrm{H}_{2}}=8 \times 10^{-2}$ mbar, $p_{\mathrm{H}_{2}} / p_{\mathrm{D}_{2}}=1.3(\triangle)$. The dashed line is the squared ratio of nuclear overlap integrals $\left|C^{\mathrm{D}_{2}}(\tau) / C^{\mathrm{H}_{2}}(\tau)\right|^{2}$ from our full quantum mechanical calculation. The harmonic order-excursion time mapping is taken from an SFA calculation for Ar at an intensity of $1.2 \times 10^{14} \mathrm{~W} \mathrm{~cm}^{-2}$, shown in figure 4a.

lowered the backing pressure until this distortion completely disappeared to minimize reabsorption of the harmonics. In this case, we read $\approx 5 \times 10^{-2}$ mbar residual pressure in the $\mathrm{HHG}$ chamber. With $\mathrm{D}_{2}$, even when the residual pressure reading is the same as for $\mathrm{H}_{2}$ in the HHG chamber, we never observed such distortions. Due to its larger weight it is pumped somewhat more efficiently than $\mathrm{H}_{2}$ in the differential pumping stage halfway between generating gas jet and MBES.

Figure 3 shows $\mathrm{D}_{2} / \mathrm{H}_{2}$ intensity ratios measured in the same generating conditions except for the gas pressures. The harmonic orders have been associated to electron excursion times as calculated with the SFA for argon, shown by the dashed line in figure 4a. With the same residual pressure readings for $\mathrm{H}_{2}$ and $\mathrm{D}_{2}$, we reproduce reasonably well for the plateau harmonics 17 to 27 the ratio of nuclear correlation functions $\left|C^{\mathrm{D}_{2}}(\tau) / C^{\mathrm{H}_{2}}(\tau)\right|^{2}$ from the full quantum mechanical calculation described in section 2.2. We compare to the theory neglecting two-centre interference because only then is the calculation independent of experimental parameters, in particular the intensity. This leads to a small under-estimation of the slope in the theoretical ratio, which is compatible with our observation. The agreement shows that we have found conditions giving approximately equal jet densities. When the $\mathrm{D}_{2}$ backing pressure is reduced such that the residual pressure readings for the two isotopes give $p_{\mathrm{H}_{2}} / p_{\mathrm{D}_{2}}=1.3$, we still obtain an increasing intensity ratio but at too low absolute values. Assuming phase matched HHG and thus a quadratic dependence of HHG intensity on the medium density, the unequal experimental densities can be corrected for by multiplying the $\mathrm{D}_{2}$ spectrum by $(1.3)^{2}$. Indeed, this correction brings the measured intensity ratio to an agreement with theory, which also shows that re-absorption within our thin gas jet is 

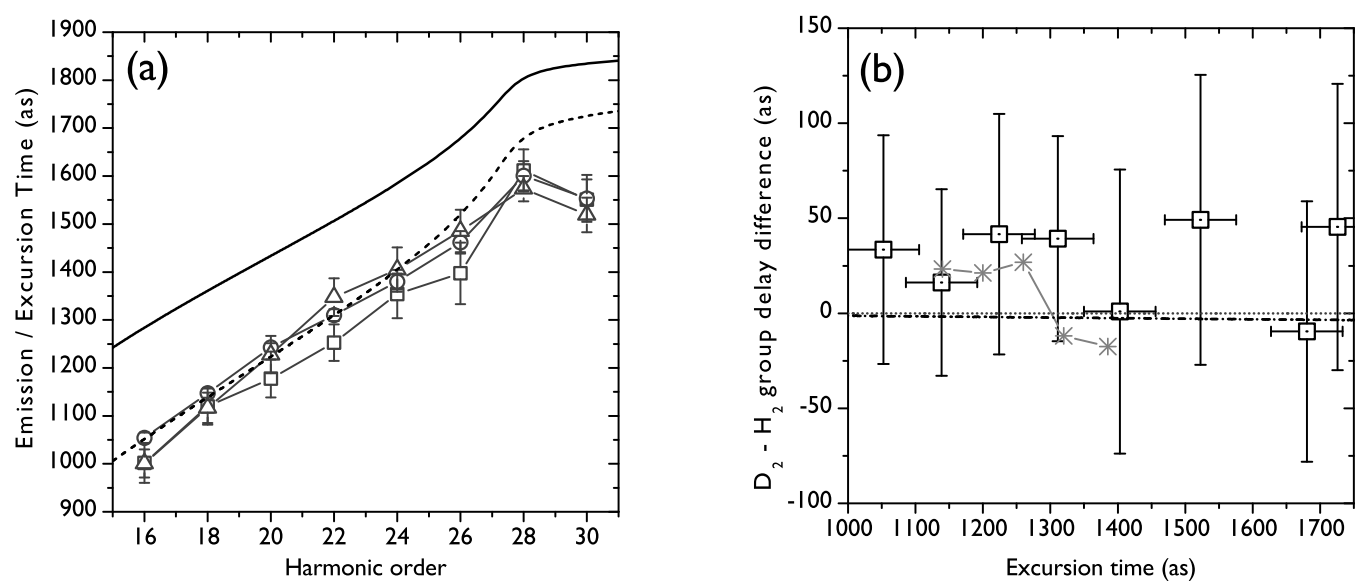

Figure 4. (a) Measured group delay or emission times for $\mathrm{H}_{2}(\square), \mathrm{D}_{2}(\mathrm{O})$ and $\operatorname{Ar}(\Delta)$. The error bars represent the accuracy of the $\tau_{\mathrm{g}}$-determination (standard deviation of the phase within the FWHM of the $2 \omega_{0}$ peak in the RABITT trace Fourier transform). The full and dashed line show recollision and excursion times, respectively, as calculated with the SFA for Ar and an intensity of $1.2 \times 10^{14} \mathrm{~W} \mathrm{~cm}^{-2}$. (b) Group Delay Differences $\Delta \tau_{\mathrm{g}}$, plotted as function of the excursion time according to the $q-\tau$ mapping from the SFA calculation shown in figure 4a. Squares are mean values over three measurements. Vertical error bars are given by the span covered by these three measurements. Stars mark results from Kanai et al.[20], scaled to correct for the different $q-\tau$ mappings (see text). The dash-dotted line is the theoretical group delay difference, derived from the full quantum mechanical treatment of the nuclear dynamics (see text).

negligible. However, such a simple correction will not be possible anymore for too high residual pressure in the vacuum chambers because reabsorption on the way from the source of the harmonics to the detector becomes important. We expect this to be the case when the $\mathrm{H}_{2}$ residual pressure causes traces of $\mathrm{H}_{2}^{+}$ions in the MBES spectra. The photoionization cross sections [35] of $\mathrm{H}_{2}$ and $\mathrm{D}_{2}$ decrease from $\approx 3.7 \mathrm{Mbarn}$ at harmonic 17 to $\approx 1$ Mbarn at harmonic 27 . For an averaged residual pressure of $10^{-2}$ mbar over 2 meters, this would result in a transmission of $T=83 \%$ for harmonic 17 and $T=95 \%$ for harmonic 27, which significantly flattens the measured spectrum as compared to the spectrum emitted at the source. As the transmission increases exponentially with decreasing density of the absorbing gas, the quicker drop of residual pressure for $D_{2}$ over the propagation distance will result in a much less pronounced flattening of the spectrum due to reabsorption. The slope of the $\mathrm{D}_{2} / \mathrm{H}_{2}$ intensity ratio obtained in these conditions will then be decreased or even inversed. We indeed measure a ratio that decreases with harmonic order (triangles in figure 3) for an $\mathrm{H}_{2}$ residual pressure reading of $p_{\mathrm{H}_{2}}=8 \times 10^{-2}$ mbar.

Figure 4 a shows the group delay measured for the three gases in the conditions of the squares in figure 3. In rare gas atoms, these group delays have been called emission times because they correspond to the real part of the recollision times calculated with the SFA [13]. This should hold in particular for argon but nevertheless there is an offset of about -250 as between the emission times given by the SFA and 
the measurement. The calculation is done for the correct effective intensity since the theoretical and experimental slopes of the emission time curves (that varies as $1 /$ intensity [13]) agree perfectly in the plateau region. We consistently observe such a shift of the measured absolute timing (reported already in [17]) and attribute it to a yet unidentified macroscopic effect in the HHG gas jet rather than physics on the single atom/molecule level. In particular, the fact that the experimental group delay curves seem to fit the theoretical excursion time curve is presumably coincidental. A striking result of figure 4 is the agreement of all three group delay curves within our error bars. We can thus conclude that the continuum electron dynamics are the same for the very light molecules and argon, which experimentally confirms the conclusion drawn from the analysis of equation (3) and validates the time-frequency mapping that is the basis of the PACER method.

For a closer analysis, we show in figure $4 \mathrm{~b}$ the difference of group delays $\Delta \tau_{\mathrm{g}}=\tau_{\mathrm{g}}^{\mathrm{D}_{2}}-\tau_{\mathrm{g}}^{\mathrm{H}_{2}}$. We plot it as a function of the excursion time, justified by the conclusion from section 2.2: the frequency-time mapping is identical for the two isotopes and any potential difference between the group delays is due to the recombination process. Different data acquisitions gave slightly varying results, differing mainly by a constant offset. We show the difference of the average over three acquisition runs for $\mathrm{D}_{2}$ (one of which is shown in figure $4 \mathrm{a}$ ) obtained in conditions of approximately equal gas density to one acquisition run for $\mathrm{H}_{2}$, shown also in figure $4 \mathrm{a}$.

Kanai et al. [20] have measured the $\mathrm{D}_{2}-\mathrm{H}_{2}$ phase difference $\Delta \varphi$ by generating harmonics in a mixture of the two gases. Their interfering emissions allow extracting the quantity $\cos (\Delta \varphi)$ from the measured spectral intensities, which leads to a sign ambiguity for $\Delta \varphi$. To compare these results to ours, we can write $\tau_{\mathrm{g}}=\partial \varphi / \partial \omega=$ $\partial \varphi / \partial \tau \times \partial \tau /\left(\omega_{0} \partial q\right)$. We then have to correct for the difference in $\partial \tau /\left(\omega_{0} \partial q\right)$ between our experiments, imposed by the different HHG intensities. In our case, $\partial \tau / \partial q \approx 44$ as in the plateau region, whereas $\partial \tau / \partial q \approx 30$ as for the conditions in [20]. We thus calculate the group delay difference from Kanai's data according to (10) and scale it by 44/30. The calculated points fall within our error margins.

We also compare to the group delay difference derived from the phase difference of the $C(\tau)$ (see (8) and figure 1) calculated in section 2.2. To this end, we take the derivative of the $\mathrm{D}_{2}-\mathrm{H}_{2}$ phase difference with respect to $\tau$ and multiply by $\partial \tau /\left(\omega_{0} \partial q\right)$ corresponding to the frequency-time mapping for our experimental conditions. The expected group delay difference is almost zero for these excursion times which is within our error margins.

\section{Conclusions}

We experimentally confirmed that the time-frequency mapping at the heart of the PACER technique is identical for $\mathrm{H}_{2}$ and $\mathrm{D}_{2}$ molecules as well as for argon atoms, which have almost the same ionization potential. This is supported by our theoretical analysis, suggesting that there is no influence of the nuclear dynamics on the recolliding 
continuum electron wavepacket.

The error bars in our current measurements do not yet allow to discern the influence of the nuclear dynamics in the $\mathrm{H}_{2}^{+}$molecule on the high harmonic phase. Future measurements with improved accuracy should allow a more precise comparison of experiment and theory. These improvements could be gained by RABITT scans over a longer delay range and more precise control over experimental parameters such as the gas density in the generating jet.

\section{Acknowledgments}

We thank John Marangos, Yann Mairesse and Christian Cornaggia for fruitful discussions. We acknowledge financial support from the EU-FP6 program LASERLAB (RII3-CT-2003-506350), the ANR Attoscience (ANR-05-BLAN-0295) and the Engineering and Physical Sciences Research Council UK (grant no. EP/C530772/2).

\section{References}

[1] J. Itatani, J. Levesque, D. Zeidler, Hiromichi Niikura, H. Pepin, J. C. Kieffer, P. B. Corkum, and D. M. Villeneuve. Tomographic imaging of molecular orbitals. Nature, 432(7019):867-871, December 2004.

[2] Thomas Schultz, Elena Samoylova, Wolfgang Radloff, Ingolf V. Hertel, Andrzej L. Sobolewski, and Wolfgang Domcke. Efficient deactivation of a model base pair via excited-state hydrogen transfer. Science, 306(5702):1765-1768, December 2004.

[3] Andrzej L. Sobolewski, Wolfgang Domcke, and C. Hättig. Tautomeric selectivity of the excitedstate lifetime of guanine/cytosine base pairs: The role of electron-driven proton-transfer processes. Proceedings of the National Academy of Sciences of the United States of America, 102(50):17903-17906, December 2005.

[4] R. Kaiser, N. Balucani, D. Charkin, and A. Mebel. A crossed beam and ab initio study of the $\mathrm{C}_{2}\left(\mathrm{X}^{1} \Sigma_{g}^{+} / \mathrm{a}^{3} \Pi_{u}\right)+\mathrm{C}_{2} \mathrm{H}_{2}\left(\mathrm{X}^{1} \Sigma_{g}^{+}\right)$reactions. Chemical Physics Letters, 382(1-2):112-119, November 2003.

[5] Alexei N. Markevitch, Dmitri A. Romanov, Stanley M. Smith, and Robert J. Levis. Rapid proton transfer mediated by a strong laser field. Physical Review Letters, 96(16):163002, 2006.

[6] Akiyoshi Hishikawa, Akitaka Matsuda, Mizuho Fushitani, and Eiji J. Takahashi. Visualizing recurrently migrating hydrogen in acetylene dication by intense ultrashort laser pulses. Physical Review Letters, 99(25):258302, 2007.

[7] Akiyoshi Hishikawa, Akitaka Matsuda, Eiji J. Takahashi, and Mizuho Fushitani. Acetylenevinylidene isomerization in ultrashort intense laser fields studied by triple ion-coincidence momentum imaging. The Journal of Chemical Physics, 128(8):084302, 2008.

[8] Hiromichi Niikura, F. Légaré, R. Hasbani, Misha Y. Ivanov, D. Villeneuve, and P. Corkum. Probing molecular dynamics with attosecond resolution using correlated wave packet pairs. Nature, 421(6925):826-829, 2003.

[9] S. Baker, J. S. Robinson, C. A. Haworth, H. Teng, R. A. Smith, C. C. Chirilă, M. Lein, J. W. G. Tisch, and J. P. Marangos. Probing proton dynamics in molecules on an attosecond time scale. Science, 312(5772):424-427, April 2006.

[10] Manfred Lein. Attosecond probing of vibrational dynamics with high-harmonic generation. Physical Review Letters, 94(5):053004, 2005.

[11] K. J. Schafer, Baorui Yang, L. F. DiMauro, and K. C. Kulander. Above threshold ionization beyond the high harmonic cutoff. Physical Review Letters, 70(11):1599-1602, March 1993. 
[12] P. B. Corkum. Plasma perspective on strong field multiphoton ionization. Physical Review Letters, 71(13):1994-1997, 1993.

[13] Y. Mairesse, A. de Bohan, L. J. Frasinski, H. Merdji, L. C. Dinu, P. Monchicourt, P. Breger, M. Kovacev, R. Taieb, B. Carré, H. G. Muller, P. Agostini, and P. Salières. Attosecond synchronization of high-harmonic soft x-rays. Science, 302(5650):1540-1543, 2003.

[14] M. Lewenstein, Ph. Balcou, Misha Y. Ivanov, Anne L'Huillier, and P. B. Corkum. Theory of high-harmonic generation by low-frequency laser fields. Physical Review A, 49(3):2117-2132, March 1994.

[15] H. Wabnitz, Y. Mairesse, L. J. Frasinski, M. Stankiewicz, W. Boutu, P. Breger, P. Johnsson, H. Merdji, P. Monchicourt, P. Salières, K. Varjú, M. Vitteau, and B. Carré. Generation of attosecond pulses in molecular nitrogen. The European Physical Journal D, 40(2):305-311, 2006.

[16] M. Lein, N. Hay, R. Velotta, J. P. Marangos, and P. L. Knight. Role of the intramolecular phase in high-harmonic generation. Phys. Rev. Lett., 88(18):183903, April 2002.

[17] W. Boutu, S. Haessler, H. Merdji, P. Breger, G. Waters, M. Stankiewicz, L. J. Frasinski, R. Taïeb, J. Caillat, A. Maquet, P. Monchicourt, B. Carré, and P. Salières. Coherent control of attosecond emission from aligned molecules. Nature Physics, 4(7):545-549, 2008.

[18] Valérie Véniard, Richard Taïeb, and Alfred Maquet. Phase dependence of (n+1)-color (n\&gt;1) ir-uv photoionization of atoms with higher harmonics. Physical Review A, 54(1):721-728, July 1996.

[19] P. M. Paul, E. S. Toma, P. Breger, G. Mullot, F. Auge, Ph Balcou, H. G. Muller, and P. Agostini. Observation of a train of attosecond pulses from high harmonic generation. Science, 292(5522):1689-1692, June 2001.

[20] Tsuneto Kanai, Eiji Takahashi, Yasuo Nabekawa, and Katsumi Midorikawa. Observing the attosecond dynamics of nuclear wavepackets in molecules by using high harmonic generation in mixed gases. New Journal of Physics, 10(2):025036, 2008.

[21] Ciprian C. Chirilă and Manfred Lein. Influence of nuclear vibration on harmonic generation in molecules. Journal of Physics B: Atomic, Molecular and Optical Physics, 39(13):S437-S444, 2006.

[22] X. Urbain, B. Fabre, E. M. Staicu-Casagrande, N. de Ruette, V. M. Andrianarijaona, J. Jureta, J. H. Posthumus, A. Saenz, E. Baldit, and C. Cornaggia. Intense-laser-field ionization of molecular hydrogen in the tunneling regime and its effect on the vibrational excitation of $\mathrm{h}_{2}^{+}$. Physical Review Letters, 92(16):163004, April 2004.

[23] C. C. Chirilă and M. Lein. High-order harmonic generation in vibrating molecules. Journal of Modern Optics, 53(1):113-124, 2006.

[24] J. H. Posthumus. The dynamics of small molecules in intense laser fields. Reports on Progress in Physics, 67(5):623-665, 2004.

[25] B. Feuerstein, Th, A. Rudenko, K. Zrost, C. D. Schröter, R. Moshammer, J. Ullrich, T. Niederhausen, and U. Thumm. Complete characterization of molecular dynamics in ultrashort laser fields. Physical Review Letters, 99(15):153002, 2007.

[26] C. C. Chirilă and M. Lein. Effect of dressing on high-order harmonic generation in vibrating $\mathrm{h}_{2}$ molecules. Physical Review A, 77(4):043403, 2008.

[27] S. Baker, J. S. Robinson, M. Lein, C. C. Chirilă, R. Torres, H. C. Bandulet, D. Comtois, J. C. Kieffer, D. M. Villeneuve, J. W. G. Tisch, and J. P. Marangos. Dynamic two-center interference in high-order harmonic generation from molecules with attosecond nuclear motion. Physical Review Letters, 101(5):053901, 2008.

[28] D. Shiner, J. M. Gilligan, B. M. Cook, and W. Lichten. $\mathrm{H}_{2}, \mathrm{D}_{2}$, and HD ionization potentials by accurate calibration of several iodine lines. Physical Review A, 47(5):4042-4045, May 1993.

[29] Misha Y. Ivanov, Thomas Brabec, and Neal Burnett. Coulomb corrections and polarization effects in high-intensity high-harmonic emission. Physical Review A, 54(1):742-745, July 1996.

[30] Y. Mairesse, A. de Bohan, L. J. Frasinski, H. Merdji, L. C. Dinu, P. Monchicourt, P. Breger, 
M. Kovačev, T. Auguste, B. Carré, H. G. Muller, P. Agostini, and P. Salières. Optimization of attosecond pulse generation. Physical Review Letters, 93(16):163901, October 2004.

[31] P. Kruit and F. H. Read. Magnetic field paralleliser for 2\&pi; electron-spectrometer and electronimage magnifier. Journal of Physics E: Scientific Instruments, 16(4):313-324, 1983.

[32] L. C. Dinu, H. G. Muller, S. Kazamias, G. Mullot, F. Augé, Ph Balcou, P. M. Paul, M. Kovacev, P. Breger, and P. Agostini. Measurement of the subcycle timing of attosecond xuv bursts in high-harmonic generation. Phys. Rev. Lett., 91(6):063901, 2003.

[33] P. W. Dooley, I. V. Litvinyuk, Kevin F. Lee, D. M. Rayner, M. Spanner, D. M. Villeneuve, and P. B. Corkum. Direct imaging of rotational wave-packet dynamics of diatomic molecules. Physical Review A, 68(2):023406, 2003.

[34] W. H. Press, B. P. Flannery, S. A. Teukolsky, and W. T. Vetterling. Numerical Recipes. Cambridge University Press, Cambridge, England, 2nd edition, 1992.

[35] C. J. Latimer, K. F. Dunn, F. P. O'Neill, M. A. Macdonald, and N. Kouchi. Photoionization of hydrogen and deuterium. The Journal of Chemical Physics, 102(2):722-725, 1995. 